\title{
New Difference Sequence Spaces Defined by Musielak-Orlicz Function
}

\author{
M. Mursaleen, ${ }^{1}$ Sunil K. Sharma, ${ }^{2}$ S. A. Mohiuddine, ${ }^{3}$ and A. Kılıçman ${ }^{4}$ \\ ${ }^{1}$ Department of Mathematics, Aligarh Muslim University, Aligarh 202002, India \\ ${ }^{2}$ Department of Mathematics, Model Institute of Engineering \& Technology, Kot Bhalwal, Jammu and Kashmir 181122, India \\ ${ }^{3}$ Department of Mathematics, Faculty of Science, King Abdulaziz University, P.O. Box 80203, Jeddah 21589, Saudi Arabia \\ ${ }^{4}$ Department of Mathematics, University Putra Malaysia (UPM), 43400 Serdang, Selangor, Malaysia
}

Correspondence should be addressed to A. Kılıçman; akilic@upm.edu.my

Received 17 March 2014; Revised 11 July 2014; Accepted 11 July 2014; Published 22 July 2014

Academic Editor: Feyzi Başar

Copyright (c) 2014 M. Mursaleen et al. This is an open access article distributed under the Creative Commons Attribution License, which permits unrestricted use, distribution, and reproduction in any medium, provided the original work is properly cited.

We introduce new sequence spaces by using Musielak-Orlicz function and a generalized $B_{\wedge}^{\mu}$-difference operator on $n$-normed space. Some topological properties and inclusion relations are also examined.

\section{Introduction and Preliminaries}

The notion of the difference sequence space was introduced by Kızmaz [1]. It was further generalized by Et and Çolak [2] as follows: $Z\left(\Delta^{\mu}\right)=\left\{x=\left(x_{k}\right) \in \omega:\left(\Delta^{\mu} x_{k}\right) \in z\right\}$ for $z=\ell_{\infty}, c$, and $c_{0}$, where $\mu$ is a nonnegative integer and

$$
\Delta^{\mu} x_{k}=\Delta^{\mu-1} x_{k}-\Delta^{\mu-1} x_{k+1}, \quad \Delta^{0} x_{k}=x_{k} \quad \forall k \in \mathbb{N}
$$

or equivalent to the following binomial representation:

$$
\Delta^{\mu} x_{k}=\sum_{v=0}^{\mu}(-1)^{v}\left(\begin{array}{c}
\mu \\
v
\end{array}\right) x_{k+v}
$$

These sequence spaces were generalized by Et and Basarir [3] taking $z=\ell_{\infty}(p), c(p)$, and $c_{0}(p)$.

Dutta [4] introduced the following difference sequence spaces using a new difference operator:

$$
Z\left(\Delta_{(\eta)}\right)=\left\{x=\left(x_{k}\right) \in \omega: \Delta_{(\eta)} x \in z\right\} \quad \text { for } z=\ell_{\infty}, c, c_{0},
$$

where $\Delta_{(\eta)} x=\left(\Delta_{(\eta)} x_{k}\right)=\left(x_{k}-x_{k-\eta}\right)$ for all $k, \eta \in \mathbb{N}$.

In [5], Dutta introduced the sequence spaces $\bar{c}\left(\|\cdot, \cdot\|, \Delta_{(\eta)}^{\mu}, p\right), \overline{c_{0}}\left(\|\cdot, \cdot\|, \Delta_{(\eta)}^{\mu}, p\right), \quad \ell_{\infty}\left(\|\cdot, \cdot\|, \Delta_{(\eta)}^{\mu}, p\right), \quad m(\|\cdot, \cdot\|$, $\left.\Delta_{(\eta)}^{\mu}, p\right)$, and $m_{0}\left(\|\cdot \cdot \cdot\|, \Delta_{(\eta)}^{\mu}, p\right)$, where $\eta, \mu \in \mathbb{N}$ and
$\Delta_{(\eta)}^{\mu} x_{k}=\left(\Delta_{(\eta)}^{\mu} x_{k}\right)=\left(\Delta_{(\eta)}^{\mu-1} x_{k}-\Delta_{(\eta)}^{\mu-1} x_{k-\eta}\right)$ and $\Delta_{(\eta)}^{0} x_{k}=x_{k}$ for all $k, \eta \in \mathbb{N}$, which is equivalent to the following binomial representation:

$$
\Delta_{(\eta)}^{\mu} x_{k}=\sum_{v=0}^{\mu}(-1)^{v}\left(\begin{array}{c}
\mu \\
v
\end{array}\right) x_{k-\eta v}
$$

The difference sequence spaces have been studied by authors [6-14] and references therein. Başar and Altay [15] introduced the generalized difference matrix $B=\left(b_{m k}\right)$ for all $k, m \in \mathbb{N}$, which is a generalization of $\Delta_{(1)}$-difference operator by

$$
b_{m k}= \begin{cases}r, & k=m \\ s, & k=m-1 \\ 0, & (k>m) \text { or }(0 \leq k<m-1) .\end{cases}
$$

Başarir and Kayikçi [16] defined the matrix $B^{\mu}\left(b_{m k}^{\mu}\right)$ which reduced the difference matrix $\Delta_{(1)}^{\mu}$ in case $r=1, s=-1$. The generalized $B^{\mu}$-difference operator is equivalent to the following binomial representation:

$$
B^{\mu} x=B^{\mu}\left(x_{k}\right)=\sum_{v=0}^{\mu}\left(\begin{array}{c}
\mu \\
v
\end{array}\right) r^{\mu-v} s^{v} x_{k-v} .
$$


Let $\wedge=\left(\wedge_{k}\right)$ be a sequence of nonzero scalars. Then, for a sequence space $E$, the multiplier sequence space $E_{\wedge}$, associated with the multiplier sequence $\wedge$, is defined as

$$
E_{\wedge}=\left\{x=\left(x_{k}\right) \in \omega:\left(\wedge_{k} x_{k}\right) \in E\right\} .
$$

An Orlicz function $M$ is a function, $M:[0, \infty) \rightarrow[0, \infty)$, which is continuous, nondecreasing, and convex with $M(0)=$ $0, M(x)>0$ for $x>0$, and $M(x) \rightarrow \infty$ as $x \rightarrow \infty$.

We say that an Orlicz function $M$ satsfies the $\Delta_{2}$ condition if there exists $K>2$ and $x_{0} \geq 0$ such that $M(2 x) \leq$ $K M(x)$ for all $x \geq x_{0}$. The $\Delta_{2}$-condition is equivalent to $M(L x) \leq K L M(x)$ for all $x>x_{0}>0$ and for $L, K>1$.

Lindenstrauss and Tzafriri [17] used the idea of Orlicz function to define the following sequence space:

$$
\ell_{M}=\left\{x \in \omega: \sum_{k=1}^{\infty} M\left(\frac{\left|x_{k}\right|}{\rho}\right)<\infty\right\}
$$

which is called an Orlicz sequence space. The space $\ell_{M}$ is a Banach space with the norm

$$
\|x\|=\inf \left\{\rho>0: \sum_{k=1}^{\infty} M\left(\frac{\left|x_{k}\right|}{\rho}\right) \leq 1\right\} .
$$

It is shown in [17] that every Orlicz sequence space $\ell_{M}$ contains a subspace isomorphic to $\ell_{p}(p \geq 1)$.

A sequence $\mathscr{M}=\left(M_{k}\right)$ of Orlicz function is called a Musielak-Orlicz function; see $[18,19]$. A sequence $\mathcal{N}=\left(N_{k}\right)$ defined by

$$
N_{k}(v)=\sup \left\{|v| u-M_{k}(u): u \geq 0\right\}, \quad k=1,2, \ldots,
$$

is called the complimentary function of a Musielak-Orlicz function $\mathscr{M}$. For a given Musielak-Orlicz function $\mathscr{M}$, the Musielak-Orlicz sequence space $t_{\mathscr{M}}$ and its subspace $h_{\mathscr{M}}$ are defined as follows:

$$
\begin{gathered}
t_{\mathscr{M}}=\left\{x \in \omega: I_{M}(c x)<\infty \text { for some } c>0\right\}, \\
h_{\mathscr{M}}=\left\{x \in \omega: I_{M}(c x)<\infty \forall c>0\right\},
\end{gathered}
$$

where $I_{\mathscr{M}}$ is a convex modular defined by

$$
I_{\mathscr{M}}(x)=\sum_{k=1}^{\infty} M_{k}\left(x_{k}\right), \quad x=\left(x_{k}\right) \in t_{M} .
$$

We consider $t_{\mathscr{M}}$ equipped with the Luxemburg norm

$$
\|x\|=\inf \left\{k>0: I_{M}\left(\frac{x}{k}\right) \leq 1\right\}
$$

or equipped with the Orlicz norm

$$
\|x\|^{0}=\inf \left\{\frac{1}{k}\left(1+I_{M}(k x)\right): k>0\right\} .
$$

By a lacunary sequence $\theta=\left(i_{r}\right), r=0,1,2, \ldots$, where $i_{0}=0$, we will mean an increasing sequence of nonnegative integers $h_{r}=\left(i_{r}-r_{r-1}\right) \rightarrow \infty(r \rightarrow \infty)$. The intervals determined by $\theta$ are denoted by $I_{r}=\left(i_{r-1}, i_{r}\right]$ and the ratio $i_{r} / i_{r-1}$ will be denoted by $q_{r}$. The space of lacunary strongly convergent sequences $N_{\theta}$ was defined by Freedman et al. [20] as follows:

$$
N_{\theta}=\left\{x=\left(x_{k}\right): \lim _{r \rightarrow \infty} \frac{1}{h_{r}} \sum_{k \in I_{r}}\left|x_{k}-L\right|=0 \text {, for some } L\right\} \text {. }
$$

The concept of 2-normed spaces was initially developed by Gähler [21] in the mid of 1960's, while that of n-normed spaces one can see in Misiak [22]. Since then, many others have studied this concept and obtained various results; see Gunawan [23, 24] and Gunawan and Mashadi [25]. For more details about sequence spaces see [26-33] and references therein. Let $n \in \mathbb{N}$ and $X$ be linear space over the field $\mathbb{K}$, where $\mathbb{K}$ is the field of real or complex numbers of dimension $d$, where $d \geq n \geq 2$.

A real valued function $\|\cdot, \ldots, \cdot\|$ on $X^{n}$ satisfying the following four conditions:

(1) $\left\|\left(x_{1}, x_{2}, \ldots, x_{n}\right)\right\|=0$ if and only if $x_{1}, x_{2}, x_{3}, \ldots, x_{n}$ are linearly dependent in $X$;

(2) $\left\|\left(x_{1}, x_{2}, \ldots, x_{n}\right)\right\|$ is invariant under permutation;

(3) $\left\|\left(\alpha x_{1}, x_{2}, \ldots, x_{n}\right)\right\|=|\alpha|\left\|\left(x_{1}, x_{2}, \ldots, x_{n}\right)\right\|$ for any $\alpha \epsilon$ $\mathbb{K}$;

(4) $\left\|\left(x+x^{\prime}, x_{2}, \ldots, x_{n}\right)\right\| \leq\left\|\left(x, x_{2}, \ldots, x_{n}\right)\right\|+$ $\left\|\left(x^{\prime}, x_{2}, \ldots, x_{n}\right)\right\|$

is called an $n$-norm on $X$ and the pair $(X,\|\cdot, \ldots, \cdot\|)$ is called an $n$-normed space over the field $\mathbb{K}$. For example, we may take $X=\mathbb{R}^{n}$ being equipped with the Euclidean $n$ norm $\left\|\left(x_{1}, x_{2}, \ldots, x_{n}\right)\right\|_{E}=$ the volume of the $n$-dimensional parallelepiped spanned by the vectors $x_{1}, x_{2}, \ldots, x_{n}$ which may be given explicitly by the formula

$$
\left\|\left(x_{1}, x_{2}, \ldots, x_{n}\right)\right\|_{E}=\left|\operatorname{det}\left(x_{i j}\right)\right|,
$$

where $x_{i}=\left(x_{1}, x_{2}, x_{3}, \ldots, x_{n}\right) \in \mathbb{R}^{n}$ for each $i=1,2,3, \ldots, n$ and $\|\cdot\|_{E}$ denotes the Euclidean norm. Let $(X,\|\cdot, \ldots, \cdot\|)$ be an $n$-normed space of dimension $d \geq n \geq 2$ and $\left\{a_{1}, a_{2}, \ldots, a_{n}\right\}$ linearly independent set in $X$. Then the following function $\|(\cdot, \ldots, \cdot)\|_{\infty}$ on $X^{n-1}$ defined by

$$
\begin{aligned}
& \left\|\left(x_{1}, x_{2}, \ldots, x_{n}\right)\right\|_{\infty} \\
& \quad=\max \left\{\left\|\left(x_{1}, x_{2}, \ldots, x_{n-1}, a_{i}\right)\right\|: i=1,2, \ldots, n\right\}
\end{aligned}
$$

defines an $(n-1)$ norm on $X$ with respect to $\left\{\left(a_{1}, a_{2}, \ldots, a_{n}\right)\right\}$.

A sequence $\left(x_{k}\right)$ in an $n$-normed space $(X,\|\cdot, \ldots, \cdot\|)$ is said to converge to some $L \in X$ if

$$
\begin{array}{r}
\lim _{k \rightarrow \infty}\left\|\left(x_{k}-L, z_{1}, \ldots, z_{n-1}\right)\right\|=0, \\
\quad \text { for every } z_{1}, \ldots, z_{n-1} \in X .
\end{array}
$$

A sequence $\left(x_{k}\right)$ in a normed space $(X,\|\cdot, \ldots, \cdot\|)$ is said to be Cauchy if

$$
\begin{aligned}
\lim _{\substack{k \rightarrow \infty \\
p \rightarrow \infty}}\left\|\left(x_{k}-x_{p}, z_{1}, \ldots, z_{n-1}\right)\right\| & =0, \\
& \text { for every } z_{1}, \ldots, z_{n-1} \in X .
\end{aligned}
$$


If every Cauchy sequence in $X$ converges to some $L \in X$ then $X$ is said to be complete with respect to the $n$-norm. Any complete $n$-normed space is said to be $n$-Banach space.

Let $(X,\|\cdot, \ldots, \cdot\|)$ be an $n$-normed space and let $s(\omega-x)$ denote the space of $X$-valued sequences. Let $p=\left(p_{k}\right)$ be any bounded sequence of positive real numbers and $\mathscr{M}=\left(M_{k}\right)$ a Musielak-Orlicz function. We define the following sequence spaces in this paper:

$$
\begin{aligned}
& w_{0}^{\theta}\left(\mathscr{M}, B_{\wedge}^{\mu}, p,\|\cdot, \ldots, \cdot\|\right) \\
& =\left\{x=\left(x_{k}\right) \in s(w-x): \lim _{r \rightarrow \infty} \frac{1}{h_{r}}\right. \\
& \left.\quad \times \sum_{k \in I_{r}} M_{k}\left(\left\|\left(\frac{B_{\wedge}^{\mu} x_{k}}{\rho}, z_{1}, \ldots, z_{n-1}\right)\right\|\right)^{p_{k}}=0, \rho>0\right\},
\end{aligned}
$$$$
w^{\theta}\left(\mathscr{M}, B_{\wedge}^{\mu}, p,\|\cdot, \ldots, \cdot\|\right)
$$$$
=\left\{x=\left(x_{k}\right) \in s(w-x): \lim _{r \rightarrow \infty} \frac{1}{h_{r}}\right.
$$$$
\times \sum_{k \in I_{r}} M_{k}\left(\left\|\left(\frac{B_{\wedge}^{\mu} x_{k}-L}{\rho}, z_{1}, \ldots, z_{n-1}\right)\right\|\right)^{p_{k}}=0,
$$$$
\text { for some } L, \rho>0\} \text {, }
$$$$
w_{\infty}^{\theta}\left(M, B_{\wedge}^{\mu}, p,\|\cdot, \ldots, \cdot\|\right)
$$$$
=\left\{x=\left(x_{k}\right) \in s(w-x): \lim _{r \rightarrow \infty} \frac{1}{h_{r}}\right.
$$$$
\left.\times \sum_{k \in I_{r}} M_{k}\left(\left\|\left(\frac{B_{\wedge}^{\mu} x_{k}}{\rho}, z_{1}, \ldots, z_{n-1}\right)\right\|\right)^{p_{k}}<\infty, \rho>0\right\} ;
$$

when $\mathscr{M}(x)=x$, we get

$$
\begin{aligned}
& w_{0}^{\theta}\left(B_{\wedge}^{\mu}, p,\|\cdot, \ldots, \cdot\|\right) \\
& =\left\{x=\left(x_{k}\right) \in s(w-x): \lim _{r \rightarrow \infty} \frac{1}{h_{r}}\right. \\
& \left.\quad \times \sum_{k \in I_{r}}\left(\left\|\left(\frac{B_{\wedge}^{\mu} x_{k}}{\rho}, z_{1}, \ldots, z_{n-1}\right)\right\|\right)^{p_{k}}=0, \rho>0\right\},
\end{aligned}
$$

$$
\begin{aligned}
& w^{\theta}\left(B_{\wedge}^{\mu}, p,\|\cdot, \ldots, \cdot\|\right) \\
& =\left\{x=\left(x_{k}\right) \in s(w-x): \lim _{r \rightarrow \infty} \frac{1}{h_{r}}\right. \\
& \quad \times \sum_{k \in I_{r}}\left(\left\|\left(\frac{B_{\wedge}^{\mu} x_{k}-L}{\rho}, z_{1}, \ldots, z_{n-1}\right)\right\|\right)^{p_{k}}=0, \\
& \quad \text { for some } L, \rho>0\}, \\
& \left.w_{\infty}^{\theta}\left(B_{\wedge}^{\mu}, p,\|\cdot, \ldots, \cdot\|\right)\right) \\
& =\left\{x=\left(x_{k}\right) \in s(w-x): \lim _{r \rightarrow \infty} \frac{1}{h_{r}}\right. \\
& \left.\quad \times \sum_{k \in I_{r}}\left(\left\|\left(\frac{B_{\wedge}^{\mu} x_{k}}{\rho}, z_{1}, \ldots, z_{n-1}\right)\right\|\right)^{p_{k}}<\infty, \rho>0\right\} ;
\end{aligned}
$$

when $p_{k}=1$, for all $k$, we get

$$
\begin{aligned}
& w_{0}^{\theta}\left(\mathscr{M}, B_{\wedge}^{\mu},\|\cdot, \ldots, \cdot\|\right) \\
& =\left\{x=\left(x_{k}\right) \in w(s-x): \lim _{r \rightarrow \infty} \frac{1}{h_{r}}\right. \\
& \left.\quad \times \sum_{k \in I_{r}} M_{k}\left(\left\|\left(\frac{B_{\wedge}^{\mu} x_{k}}{\rho}, z_{1}, \ldots, z_{n-1}\right)\right\|\right)=0, \rho>0\right\}, \\
& w^{\theta}\left(\mathscr{M}, B_{\wedge}^{\mu},\|\cdot, \ldots, \cdot\|\right) \\
& =\left\{\begin{array}{c}
x=\left(x_{k}\right) \in w(s-x): \lim _{r \rightarrow \infty} \frac{1}{h_{r}} \\
\quad \times \sum_{k \in I_{r}} M_{k}\left(\left\|\left(\frac{B_{\wedge}^{\mu} x_{k}-L}{\rho}, z_{1}, \ldots, z_{n-1}\right)\right\|\right)=0,
\end{array}\right.
\end{aligned}
$$$$
\text { for some } L, \rho>0\} \text {, }
$$

$$
\begin{aligned}
& w_{\infty}^{\theta}\left(\mathscr{M}, B_{\wedge}^{\mu},\|\cdot, \ldots, \cdot\|\right) \\
& =\left\{x=\left(x_{k}\right) \in w(s-x): \lim _{r \rightarrow \infty} \frac{1}{h_{r}}\right. \\
& \left.\quad \times \sum_{k \in I_{r}} M_{k}\left(\left\|\left(\frac{B_{\wedge}^{\mu} x_{k}}{\rho}, z_{1}, \ldots, z_{n-1}\right)\right\|\right)<\infty, \rho>0\right\} .
\end{aligned}
$$


The following inequality will be used throughout the paper. If $0 \leq p_{k} \leq \sup p_{k}=H, k=\max \left(1,2^{H-1}\right)$, then

$$
\left|a_{k}+b_{k}\right|^{p_{k}} \leq K\left\{\left|a_{k}\right|^{p_{k}}+\left|b_{k}\right|^{p_{k}}\right\}
$$

for all $k$ and $a_{k}, b_{k} \in \mathbb{C}$. Also $|a|^{p_{k}} \leq \max \left(1,|a|^{H}\right)$ for all $a \in \mathbb{C}$.

\section{Main Results}

Theorem 1. Let $\mathscr{M}=\left(M_{k}\right)$ be a Musielak-Orlicz function and $p=\left(p_{k}\right)$ a bounded sequence of positive real numbers; the spaces $w_{0}^{\theta}\left(\mathscr{M}, B_{\wedge}^{\mu}, p,\|\cdot, \ldots, \cdot\|\right), w^{\theta}\left(\mathscr{M}, B_{\wedge}^{\mu}, p,\|\cdot, \ldots, \cdot\|\right)$, and $w_{\infty}^{\theta}\left(\mathscr{M}, B_{\wedge}^{\mu}, p,\|\cdot, \ldots, \cdot\|\right)$ are linear over the field of complex numbers $\mathbb{C}$.

Proof. Let $x=\left(x_{k}\right), y=\left(y_{k}\right) \in w_{0}^{\theta}\left(\mathscr{M}, B_{\wedge}^{\mu}, p,\|\cdot, \ldots, \cdot\|\right)$, and $\alpha, \beta \in \mathbb{C}$. Then there exist positive real numbers $\rho_{1}$ and $\rho_{2}$ such that

$$
\begin{aligned}
& \lim _{r \rightarrow \infty} \frac{1}{h_{r}} \sum_{k \in I_{r}} M_{k}\left(\left\|\left(\frac{B_{\wedge}^{\mu} x_{k}}{\rho_{1}}, z_{1}, \ldots, z_{n-1}\right)\right\|\right)^{p_{k}}=0, \\
& \lim _{r \rightarrow \infty} \frac{1}{h_{r}} \sum_{k \in I_{r}} M_{k}\left(\left\|\left(\frac{B_{\wedge}^{\mu} y_{k}}{\rho_{2}}, z_{1}, \ldots, z_{n-1}\right)\right\|\right)^{p_{k}}=0 .
\end{aligned}
$$

Define $\rho_{3}=\max \left(2|\alpha| \rho_{1}, 2|\beta| \rho_{2}\right)$. Since $\|\cdot, \ldots, \cdot\|$ is an $n$-norm on $X$ and $M_{k}^{\prime} s$ are nondecreasing and convex functions so by using inequality (23) we have

$$
\begin{aligned}
& \lim _{r \rightarrow \infty} \frac{1}{h_{r}} \sum_{k \in I_{r}} M_{k}\left(\left\|\left(\frac{B_{\wedge}^{\mu}\left(\alpha x_{k}+\beta y_{k}\right)}{\rho_{3}}, z_{1}, \ldots, z_{n-1}\right)\right\|\right)^{p_{k}} \\
& \leq \lim _{r \rightarrow \infty} \frac{1}{h_{r}} \sum_{k \in I_{r}} M_{k}\left(\left\|\left(\frac{B_{\wedge}^{\mu} \alpha x_{k}}{\rho_{3}}, z_{1}, \ldots, z_{n-1}\right)\right\|\right. \\
& \left.+\left\|\left(\frac{B_{\wedge}^{\mu} \beta y_{k}}{\rho_{3}}, z_{1}, \ldots, z_{n-1}\right)\right\|\right)^{p_{k}} \\
& \leq K_{r \rightarrow \infty} \frac{1}{h_{r}} \sum_{k \in I_{r}} \frac{1}{2^{p_{k}}} M_{k}\left(\left\|\left(\frac{B_{\wedge}^{\mu} x_{k}}{\rho_{1}}, z_{1}, \ldots, z_{n-1}\right)\right\|\right)^{p_{k}} \\
& \quad+K_{r \rightarrow \infty} \frac{1}{h_{r}} \sum_{k \in I_{r}} \frac{1}{2^{p_{k}}} M_{k}\left(\left\|\left(\frac{B_{\wedge}^{\mu} y_{k}}{\rho_{2}}, z_{1}, \ldots, z_{n-1}\right)\right\|\right)^{p_{k}} \\
& =0 .
\end{aligned}
$$

Thus, we have $\alpha x+\beta y \in w_{0}^{\theta}\left(\mathscr{M}, B_{\wedge}^{\mu}, p,\|\cdot, \ldots, \cdot\|\right)$. Hence $w_{0}^{\theta}\left(\mathscr{M}, B_{\wedge}^{\mu}, p,\|\cdot, \ldots, \cdot\|\right)$ is a linear space. Similarly, we can prove that $w^{\theta}\left(\mathscr{M}, B_{\wedge}^{\mu}, p,\|\cdot, \ldots, \cdot\|\right)$ and $w_{\infty}^{\theta}\left(\mathscr{M}, B_{\wedge}^{\mu}\right.$, $p,\|\cdot, \ldots, \cdot\|)$ are linear spaces. This completes the proof of the theorem.

Theorem 2. Let $M=\left(M_{k}\right)$ be a Musielak-Orlicz function and $p=\left(p_{k}\right)$ a bounded sequence of positive real numbers; the space $w_{0}^{\theta}\left(\mathscr{M}, B_{\wedge}^{\mu}, p,\|\cdot, \ldots, \cdot\|\right)$ is a topological linear space paranormed by

$g(x)$

$$
=\inf \left\{\rho^{p_{r} / M}:\right.
$$

$$
\begin{aligned}
& \left(\frac{1}{h_{r}} \sum_{k \in I_{r}} M_{k}\left(\left\|\left(\frac{B_{\wedge}^{\mu} x_{k}}{\rho}, z_{1}, \ldots, z_{n-1}\right)\right\|\right)^{p_{k}}\right)^{1 / M} \\
& \leq 1\},
\end{aligned}
$$

where $M=\max \left(1, \sup _{k} p_{k}<\infty\right)$.

Proof. Clearly $g(x) \geq 0$ for $x=\left(x_{k}\right) \in w_{0}^{\theta}\left(\mathscr{M}, B_{\wedge}^{\mu}\right.$, $p,\|\cdot, \ldots, \cdot\|)$. Since $M_{k}(0)=0$, we get $g(0)=0$. Again, if $g(x)=0$, then

$g(x)$

$$
\begin{aligned}
& =\inf \left\{\rho^{p_{r} / M}:\right. \\
& \left.\qquad \frac{1}{h_{r}} \sum_{k \in I_{r}} M_{k}\left(\left\|\left(\frac{B_{\wedge}^{\mu} x_{k}}{\rho}, z_{1}, \ldots, z_{n-1}\right)\right\|\right)^{p_{k}}\right)^{1 / M} \\
& \leq 1\}=0 .
\end{aligned}
$$

This implies that, for a given $\varepsilon>0$, there exist some $\rho_{\varepsilon}(0<$ $\left.\rho_{\varepsilon}<\varepsilon\right)$ such that

$$
\left(\frac{1}{h_{r}} \sum_{k \in I_{r}} M_{k}\left(\left\|\left(\frac{B_{\wedge}^{\mu} x_{k}}{\rho_{\varepsilon}}, z_{1}, \ldots, z_{n-1}\right)\right\|\right)^{p_{k}}\right)^{1 / M} \leq 1 .
$$

Thus

$$
\left(\frac{1}{h_{r}} \sum_{k \in I_{r}} M_{k}\left(\left\|\left(\frac{B_{\wedge}^{\mu} x_{k}}{\varepsilon}, z_{1}, \ldots, z_{n-1}\right)\right\|\right)^{p_{k}}\right)^{1 / M}
$$

$$
\begin{aligned}
& \leq\left(\frac{1}{h_{r}} \sum_{k \in I_{r}} M_{k}\left(\left\|\left(\frac{B_{\wedge}^{\mu} x_{k}}{\rho_{\varepsilon}}, z_{1}, \ldots, z_{n-1}\right)\right\|\right)^{p_{k}}\right)^{1 / M} \\
& \leq 1
\end{aligned}
$$

for each $r$, and suppose that $x_{k} \neq 0$ for each $k \in \mathbb{N}$. This implies that $B_{\wedge}^{\mu} x_{k} \neq 0$ for each $k \in \mathbb{N}$. Let $\varepsilon \rightarrow 0$, then

$$
\left(\left(\left\|\left(\frac{B_{\wedge}^{\mu} x_{k}}{\rho_{\varepsilon}}, z_{1}, \ldots, z_{n-1}\right)\right\|\right)^{p_{k}}\right)^{1 / M} \longrightarrow \infty ;
$$


which is a contradiction. Therefore, $B_{\wedge}^{\mu} x_{k}=0$ for each $k$ and thus $x_{k}=0$ for each $k \in \mathbb{N}$. Let $\rho_{1}>0$ and $\rho_{2}>0$ be such that

$$
\begin{aligned}
& \left(\frac{1}{h_{r}} \sum_{k \in I_{r}} M_{k}\left(\left\|\left(\frac{B_{\wedge}^{\mu} x_{k}}{\rho_{1}}, z_{1}, \ldots, z_{n-1}\right)\right\|\right)^{p_{k}}\right)^{1 / M} \leq 1, \\
& \left(\frac{1}{h_{r}} \sum_{k \in I_{r}} M_{k}\left(\left\|\left(\frac{B_{\wedge}^{\mu} y_{k}}{\rho_{2}}, z_{1}, \ldots, z_{n-1}\right)\right\|\right)^{p_{k}}\right)^{1 / M} \leq 1
\end{aligned}
$$

for each $r$.

Let $\rho=\rho_{1}+\rho_{2}$; then by using Minkowski's inequality, we have

$$
\begin{aligned}
& \left(\frac{1}{h_{r}} \sum_{k \in I_{r}} M_{k}\left(\left\|\left(\frac{B_{\wedge}^{\mu}\left(x_{k}+y_{k}\right)}{\rho}, z_{1}, \ldots, z_{n-1}\right)\right\|\right)^{p_{k}}\right)^{1 / M} \\
& \leq\left(\frac{1}{h_{r}} \sum_{k \in I_{r}} M_{k}\left(\left\|\left(\frac{B_{\wedge}^{\mu} x_{k}+B_{\wedge}^{\mu} y_{k}}{\rho_{1}+\rho_{2}}, z_{1}, \ldots, z_{n-1}\right)\right\|\right)^{p_{k}}\right)^{1 / M} \\
& \leq\left(\frac{1}{h_{r}} \sum_{k \in I_{r}} M_{k}\right. \\
& \quad \times\left(\left(\frac{\rho_{1}}{\rho_{1}+\rho_{2}}\right)\left\|\left(\frac{B_{\wedge}^{\mu} x_{k}}{\rho_{1}}, z_{1}, \ldots, z_{n-1}\right)\right\|\right. \\
& \leq\left(\frac{\rho_{1}}{\rho_{1}+\rho_{2}}\right) \\
& \left.\left.\quad \times\left(\frac{1}{h_{r}} \sum_{k \in I_{r}} M_{k}\left(\left\|\left(\frac{B_{\wedge}^{\mu} x_{k}}{\rho_{1}}, z_{1}, \ldots, z_{n-1}\right)\right\|\right)^{p_{k}}\right)\left\|\left(\frac{B_{\wedge}^{\mu} y_{k}}{\rho_{2}}, z_{1}, \ldots, z_{n-1}\right)\right\|\right)^{1 / M}\right)^{p_{k}} \\
& \quad+\left(\frac{\rho_{2}}{\rho_{1}+\rho_{2}}\right) \\
& \quad \times\left(\frac{1}{h_{r}} \sum_{k \in I_{r}} M_{k}\left(\left\|\left(\frac{B_{\wedge}^{\mu} y_{k}}{\rho_{2}}, z_{1}, \ldots, z_{n-1}\right)\right\|\right)^{p_{k}}\right)^{1 / M} \leq 1 .
\end{aligned}
$$

Since $\rho^{\prime} s$ are nonnegative, we have $g(x+y)$

$$
\begin{aligned}
=\inf \left\{\rho^{p_{r} / M}:\right. & \left(\frac{1}{h_{r}} \sum_{k \in I_{r}} M_{k}\right. \\
& \left.\times\left(\left\|\left(\frac{B_{\wedge}^{\mu} x_{k}}{\rho}, z_{1}, \ldots, z_{n-1}\right)\right\|\right)^{p_{k}}\right)^{1 / M} \\
\leq 1\} &
\end{aligned}
$$

$$
\begin{aligned}
\leq \inf \left\{\rho_{1}{ }^{p_{r} / M}:\right. & \left(\frac{1}{h_{r}} \sum_{k \in I_{r}} M_{k}\right. \\
& \left.\times\left(\left\|\left(\frac{B_{\wedge}^{\mu} x_{k}}{\rho_{1}}, z_{1}, \ldots, z_{n-1}\right)\right\|\right)^{p_{k}}\right)^{1 / M}
\end{aligned}
$$

$$
\begin{aligned}
& \leq 1\} \\
& +\inf \left\{\rho_{2}^{p_{r} / M}:\left(\frac{1}{h_{r}} \sum_{k \in I_{r}} M_{k}\right.\right. \\
& \left.\quad \times\left(\left\|\left(\frac{B_{\wedge}^{\mu} y_{k}}{\rho_{2}}, z_{1}, \ldots, z_{n-1}\right)\right\|\right)^{p_{k}}\right)^{1 / M} \\
& \leq 1\} .
\end{aligned}
$$

Therefore, $g(x+y) \leq g(x)+g(y)$.

Finally, we prove that the scalar multiplication is continuous. Let $v$ be any complex number. By definition,

$$
\begin{aligned}
& g(\nu x) \\
& =\inf \left\{\rho^{p_{r} / M}:\left(\frac{1}{h_{r}} \sum_{k \in I_{r}} M_{k}\right.\right. \\
& \left.\quad \times\left(\left\|\left(\frac{\nu B_{\wedge}^{\mu} x_{k}}{\rho}, z_{1}, \ldots, z_{n-1}\right)\right\|\right)^{p_{k}}\right)^{1 / M} \\
& \leq 1\} .
\end{aligned}
$$


Then

$$
\begin{aligned}
& g(\nu x) \\
& =\inf \left\{(|\nu| t)^{p_{r} / M}:\left(\frac{1}{h_{r}} \sum_{k \in I_{r}} M_{k}\right.\right. \\
& \left.\times\left(\left\|\left(\frac{B_{\wedge}^{\mu} x_{k}}{t}, z_{1}, \ldots, z_{n-1}\right)\right\|\right)^{p_{k}}\right)^{1 / M} \\
& \leq 1\},
\end{aligned}
$$

where $t=\rho /|\nu|$. Since $|\nu|^{p_{r}} \leq \max \left(1,|\nu| \sup p_{k}\right)$, we have

$$
\begin{aligned}
g(\nu x)= & \max \left(1,|\nu| \sup p_{k}\right) \inf \\
\times & \left\{(t)^{p_{r} / M}:\right. \\
& \left(\frac{1}{h_{r}} \sum_{k \in I_{r}} M_{k}\right. \\
& \left.\left.\quad \times\left(\left\|\left(\frac{B_{\wedge}^{\mu} x_{k}}{t}, z_{1}, \ldots, z_{n-1}\right)\right\|\right)^{p_{k}}\right)^{1 / M} \leq 1\right\} .
\end{aligned}
$$

So, the fact that scalar multiplication is continuous follows from the above inequality. This completes the proof of theorem.

Theorem 3. Let $\mathscr{M}=\left(M_{k}\right)$ be a Musielak-Orlicz function. If $\sup _{k}\left(M_{k}(x)\right)^{p_{k}}<\infty$ for all fixed $x>0$, then $w^{\theta}\left(\mathscr{M}, B_{\wedge}^{\mu}, p,\|\cdot, \ldots, \cdot\|\right) \subset w_{\infty}^{\theta}\left(\mathscr{M}, B_{\wedge}^{\mu}, p,\|\cdot, \ldots, \cdot\|\right)$.

Proof. Let $x=\left(x_{k}\right) \in w^{\theta}\left(\mathscr{M}, B_{\wedge}^{\mu}, p,\|\cdot, \ldots, \cdot\|\right)$. Then there exists some positive number $\rho_{1}$ such that

$$
\lim _{r \rightarrow \infty} \frac{1}{h_{r}} \sum_{k \in I_{r}} M_{k}\left(\left\|\left(\frac{B_{\wedge}^{\mu} x_{k}-L}{\rho_{1}}, z_{1}, \ldots, z_{n-1}\right)\right\|\right)^{p_{k}}=0
$$

Define $\rho=2 \rho_{1}$. Since $M_{k}$ is nondecreasing and convex and by using inequality (23), we have

$$
\begin{aligned}
& \lim _{r \rightarrow \infty} \frac{1}{h_{r}} \sum_{k \in I_{r}} M_{k}\left(\left\|\left(\frac{B_{\wedge}^{\mu} x_{k}}{\rho}, z_{1}, \ldots, z_{n-1}\right)\right\|\right)^{p_{k}} \\
& =\lim _{r \rightarrow \infty} \frac{1}{h_{r}} \sum_{k \in I_{r}} M_{k}\left(\left\|\left(\frac{B_{\wedge}^{\mu} x_{k}-L+L}{\rho}, z_{1}, \ldots, z_{n-1}\right)\right\|\right)^{p_{k}}
\end{aligned}
$$

$$
\begin{gathered}
\leq K\left\{\lim _{r \rightarrow \infty} \frac{1}{h_{r}} \sum_{k \in I_{r}} M_{k} \frac{1}{2^{p_{k}}}\left(\left\|\left(\frac{B_{\wedge}^{\mu} x_{k}-L}{\rho_{1}}, z_{1}, \ldots, z_{n-1}\right)\right\|\right)^{p_{k}}\right. \\
\left.\quad+\lim _{r \rightarrow \infty} \frac{1}{h_{r}} \sum_{k \in I_{r}} M_{k} \frac{1}{2^{p_{k}}}\left(\left\|\left(\frac{L}{\rho_{1}}, z_{1}, \ldots, z_{n-1}\right)\right\|\right)^{p_{k}}\right\} \\
<K\left\{\lim _{r \rightarrow \infty} \frac{1}{h_{r}} \sum_{k \in I_{r}} M_{k}\left(\left\|\left(\frac{B_{\wedge}^{\mu} x_{k}-L}{\rho_{1}}, z_{1}, \ldots, z_{n-1}\right)\right\|\right)^{p_{k}}\right. \\
\left.\quad+\lim _{r \rightarrow \infty} \frac{1}{h_{r}} \sum_{k \in I_{r}} M_{k}\left(\left\|\left(\frac{L}{\rho_{1}}, z_{1}, \ldots, z_{n-1}\right)\right\|\right)^{p_{k}}\right\} .
\end{gathered}
$$

Hence $x=\left(x_{k}\right) \in w_{\infty}^{\theta}\left(\mathscr{M}, B_{\wedge}^{\mu}, p,\|\cdot, \ldots, \cdot\|\right)$. This completes the proof of the theorem.

Theorem 4. Let $\mathscr{M}=\left(M_{k}\right)$ be a Musielak-Orlicz function and $0<h=\inf p_{k}$. Then

$$
w_{\infty}^{\theta}\left(\mathscr{M}, B_{\wedge}^{\mu}, p,\|\cdot, \ldots, \cdot\|\right) \subset w_{0}^{\theta}\left(\mathscr{M}, B_{\wedge}^{\mu}, p,\|\cdot, \ldots, \cdot\|\right)
$$

if and only if

$$
\lim _{r \rightarrow \infty} \frac{1}{h_{r}} \sum_{k \in I_{r}} M_{k}(t)^{p_{k}}=\infty, \quad \text { for some } t>0 .
$$

Proof. Let $w_{\infty}^{\theta}\left(\mathscr{M}, B_{\wedge}^{\mu}, p,\|\cdot, \ldots, \cdot\|\right) \subset w_{0}^{\theta}\left(\mathscr{M}, B_{\wedge}^{\mu}, p,\|\cdot, \ldots, \cdot\|\right)$. Suppose (40) does not hold. Therefore there are a subinterval $I_{r(m)}$ of the set of intervals $I_{r}$ and a number $n_{0}$, where $n_{0}=$ $\left\|\left(B_{\wedge}^{\mu} x_{k} / \rho, z_{1}, \ldots, z_{n-1}\right)\right\|$ for all $k$, such that

$$
\frac{1}{h_{r}(m)} \sum_{k \in I_{r(m)}} M\left(n_{0}\right)^{p_{k}} \leq N<\infty, \quad m=1,2,3, \ldots
$$

Let us define $x=\left(x_{k}\right)$ as follows:

$$
B_{\wedge}^{\mu} x_{k}= \begin{cases}\rho n_{0}, & k \in I_{r(m)} \\ 0, & k \notin I_{r(m)}\end{cases}
$$

Thus by (41), $x=\left(x_{k}\right) \in w_{\infty}^{\theta}\left(\mathscr{M}, B_{\wedge}^{\mu}, p,\|\cdot, \ldots, \cdot\|\right)$. But $x=$ $\left(x_{k}\right) \notin w_{0}^{\theta}\left(\mathscr{M}, B_{\wedge}^{\mu}, p,\|\cdot, \ldots, \cdot\|\right)$. Hence (40) must hold.

Conversely, suppose that (40) holds and let $x=\left(x_{k}\right) \in$ $w_{\infty}^{\theta}\left(\mathscr{M}, B_{\wedge}^{\mu}, p,\|\cdot, \ldots, \cdot\|\right)$. Then,

$$
\frac{1}{h_{r}} \sum_{k \in I_{r}} M_{k}\left(\left\|\left(\frac{B_{\wedge}^{\mu} x_{k}}{\rho}, z_{1}, \ldots, z_{n-1}\right)\right\|\right)^{p_{k}} \leq N<\infty .
$$

Suppose that $x=\left(x_{k}\right) \notin w_{0}^{\theta}\left(\mathscr{M}, B_{\wedge}^{\mu}, p,\|\cdot, \ldots, \cdot\|\right)$. Then for some number $\varepsilon, 1>\varepsilon>0$, there is a number $N_{0}$ such that, for a subinterval $I_{r(m)}$ of the set of intervals $I_{r}$,

$$
\left\|\left(\frac{B_{\wedge}^{\mu} x_{k}}{\rho}, z_{1}, \ldots, z_{n-1}\right)\right\|>\varepsilon \quad \text { for } N \geq N_{0} .
$$


We have $M_{k}\left(\left\|\left(B_{\wedge}^{\mu} x_{k} / \rho, z_{1}, \ldots, z_{n-1}\right)\right\|\right) \geq M(\varepsilon)^{p_{k}}$, which contradicts (40) by using (43). Hence we get

$$
w_{\infty}^{\theta}\left(\mathscr{M}, B_{\wedge}^{\mu}, p,\|\cdot, \ldots, \cdot\|\right) \subset w_{0}^{\theta}\left(\mathscr{M}, B_{\wedge}^{\mu}, p,\|\cdot, \ldots, \cdot\|\right) .
$$

This completes the proof.

Theorem 5. Let $0<h=\inf p_{k} \leq \sup p_{k}=H<\infty$. For any Musielak-Orlicz function $\mathscr{M}=\left(M_{k}\right)$ which satisfies $\Delta_{2}$ condition, one has

(i) $w_{0}^{\theta}\left(B_{\wedge}^{\mu}, p,\|\cdot, \ldots, \cdot\|\right) \subset w_{0}^{\theta}\left(\mathscr{M}, B_{\wedge}^{\mu}, p,\|\cdot, \ldots, \cdot\|\right)$

(ii) $w^{\theta}\left(B_{\wedge}^{\mu}, p,\|\cdot, \ldots, \cdot\|\right) \subset w^{\theta}\left(\mathscr{M}, B_{\wedge}^{\mu}, p,\|\cdot, \ldots, \cdot\|\right)$

(iii) $w_{\infty}^{\theta}\left(B_{\wedge}^{\mu}, p,\|\cdot, \ldots, \cdot\|\right) \subset w_{\infty}^{\theta}\left(\mathscr{M}, B_{\wedge}^{\mu}, p,\|\cdot, \ldots, \cdot\|\right)$.

Proof. (i) Let $x=\left(x_{k}\right) \in w_{0}^{\theta}\left(B_{\wedge}^{\mu}, p,\|\cdot, \ldots, \cdot\|\right)$. Then, we have

$$
\frac{1}{h_{r}} \sum_{k \in I_{r}}\left(\left\|\left(\frac{B_{\wedge}^{\mu} x_{k}}{\rho}, z_{1}, \ldots, z_{n-1}\right)\right\|\right)^{p_{k}} \longrightarrow 0 \quad \text { as } r \longrightarrow \infty .
$$

Let $\varepsilon>0$, and choose $\delta$ with $0<\delta<1$ such that $M_{k}<\varepsilon$ for $0 \leq t \leq \delta$. We can write

$$
\begin{aligned}
& \frac{1}{h_{r}} \sum_{k \in I_{r}}\left(\left\|\left(\frac{B_{\wedge}^{\mu} x_{k}}{\rho}, z_{1}, \ldots, z_{n-1}\right)\right\|\right)^{p_{k}} \\
& =\frac{1}{h_{r}} \sum_{\substack{\left\|\left(B_{\wedge}^{\mu} x_{k} / \rho, z_{1}, \ldots, z_{n-1}\right)\right\| \leq \delta\\
}}\left(\left\|\left(\frac{B_{\wedge}^{\mu} x_{k}}{\rho}, z_{1}, \ldots, z_{n-1}\right)\right\|\right)^{p_{k}} \\
& +\frac{1}{h_{r}} \sum_{\left\|\left(B_{\wedge}^{\mu} x_{k} / \rho, z_{1}, \ldots, I_{n-1}\right)\right\|>\delta}\left(\left\|\left(\frac{B_{\wedge}^{\mu} x_{k}}{\rho}, z_{1}, \ldots, z_{n-1}\right)\right\|\right)^{p_{k}} .
\end{aligned}
$$

For the first summation above, we can write

$$
\begin{aligned}
& \frac{1}{h_{r}} \sum_{\left\|\left(B_{\wedge}^{\mu} x_{k} / \rho, z_{1}, \ldots, z_{n-1}\right)\right\| \leq \delta}\left(\left\|\left(\frac{B_{\wedge}^{\mu} x_{k}}{\rho}, z_{1}, \ldots, z_{n-1}\right)\right\|\right)^{p_{k}} \\
& \quad<\max \left(\varepsilon, \varepsilon^{h}\right) .
\end{aligned}
$$

By using continuity of $M_{k}$, for the second summation we can write

$$
\left\|\left(\frac{B_{\wedge}^{\mu} x_{k}}{\rho}, z_{1}, \ldots, z_{n-1}\right)\right\|<1+\frac{\left(\left\|\left(B_{\wedge}^{\mu} x_{k} / \rho, z_{1}, \ldots, z_{n-1}\right)\right\|\right)}{\delta} .
$$

Since each $M_{k}$ is nondecreasing and convex and satisfies $\Delta_{2}$ condition, it follows that

$$
\begin{aligned}
& \frac{1}{h_{r}} \sum_{k \in I_{r}} M_{k}\left(\left\|\left(\frac{B_{\wedge}^{\mu} x_{k}}{\rho}, z_{1}, \ldots, z_{n-1}\right)\right\|\right)^{p_{k}} \\
& \leq \max \left(\varepsilon, \varepsilon^{h}\right) \\
& \quad+\max \left\{1,\left[\frac{2 M_{k}\left(\left\|\left(B_{\wedge}^{\mu} x_{k} / \rho, z_{1}, \ldots, z_{n-1}\right)\right\|\right)}{\delta}\right]^{h}\right\} \\
& \quad \times \frac{1}{h_{r}} \sum_{k \in I_{r}}\left(\left\|\left(\frac{B_{\wedge}^{\mu} x_{k}}{\rho}, z_{1}, \ldots, z_{n-1}\right)\right\|\right)^{p_{k}} .
\end{aligned}
$$

Taking limit as $\varepsilon \rightarrow 0$ and $r \rightarrow \infty$, it follows that $x=$ $\left(x_{k}\right) \in w_{0}^{\theta}\left(\mathscr{M}, B_{\wedge}^{\mu}, p,\|\cdot, \ldots, \cdot\|\right)$. Hence $w_{0}^{\theta}\left(B_{\wedge}^{\mu}, p,\|\cdot, \ldots, \cdot\|\right) \subset$ $w_{0}^{\theta}\left(\mathscr{M}, B_{\wedge}^{\mu}, p,\|\cdot, \ldots, \cdot\|\right)$. Similarly, we can prove (ii) and (iii). This completes the proof of the theorem.

Theorem 6. Let $\mathscr{M}=\left(M_{k}\right)$ be a Musielak-Orlicz function. Then the following statements are equivalent:

(i) $w_{\infty}^{\theta}\left(B_{\wedge}^{\mu}, p,\|\cdot, \ldots, \cdot\|\right) \subset w_{\infty}^{\theta}\left(\mathscr{M}, B_{\wedge}^{\mu}, p,\|\cdot, \ldots, \cdot\|\right)$;

(ii) $w_{0}^{\theta}\left(B_{\wedge}^{\mu}, p,\|\cdot, \ldots, \cdot\|\right) \subset w_{\infty}^{\theta}\left(\mathscr{M}, B_{\wedge}^{\mu}, p,\|\cdot, \ldots, \cdot\|\right)$;

(iii) $\sup _{r}\left(1 / h_{r}\right) \sum_{k \in I_{r}} M_{k}(t)^{p_{k}}<\infty$ for all $t>0$, where $t=$ $\left\|B_{\wedge}^{\mu} x_{k} / \rho, z_{1}, \ldots, z_{n-1}\right\|$.

Proof. (i) $\Rightarrow$ (ii) Suppose (i) holds. In order to prove (ii) we have to show that

$$
w^{\theta}\left(B_{\wedge}^{\mu}, p,\|\cdot \ldots, \cdot\|\right) \subset w_{\infty}^{\theta}\left(\mathscr{M}, B_{\wedge}^{\mu}, p,\|\cdot, \ldots, \cdot\|\right) .
$$

Let $x=\left(x_{k}\right) \in w_{0}^{\theta}\left(B_{\wedge}^{\mu}, p,\|\cdot, \ldots, \cdot\|\right)$. Then for a given $\varepsilon>0$ there exists $s>s_{\varepsilon}$ such that

$$
\frac{1}{h_{r}} \sum_{k \in I_{r}}\left(\left\|\left(\frac{B_{\wedge}^{\mu} x_{k}}{\rho}, z_{1}, \ldots, z_{n-1}\right)\right\|\right)^{p_{k}}<\varepsilon .
$$

Hence there exists $K>0$ such that

$$
\sup _{r} \frac{1}{h_{r}} \sum_{k \in I_{r}}\left(\left\|\left(\frac{B_{\wedge}^{\mu} x_{k}}{\rho}, z_{1}, \ldots, z_{n-1}\right)\right\|\right)^{p_{k}}<K .
$$

This shows that $x=\left(x_{k}\right) \in w_{\infty}^{\theta}\left(\mathscr{M}, B_{\wedge}^{\mu}, p,\|\cdot, \ldots, \cdot\|\right)$.

(ii) $\Rightarrow$ (iii) Suppose (ii) holds and (iii) fails to hold. Then for some $t>0$,

$$
\sup _{r} \frac{1}{h_{r}} \sum_{k \in I_{r}} M_{k}(\varepsilon)^{p_{k}}=\infty
$$

and, therefore, we can find a subinterval $I_{r(m)}$ of the set of intervals $I_{r}$ such that

$$
\frac{1}{h_{r}(m)} \sum_{k \in I_{r(m)}} M_{k}\left(\frac{1}{m}\right)^{p_{k}} \geq m, \quad m=1,2,3, \ldots
$$


Let us define $x=\left(x_{k}\right)$ as follows:

$$
B_{\wedge}^{\mu} x_{k}= \begin{cases}\frac{\rho}{m}, & k \in I_{r(m)} \\ 0, & k \notin I_{r(m)} .\end{cases}
$$

Thus $x=\left(x_{k}\right) \in w_{0}^{\theta}\left(B_{\wedge}^{\mu}, p,\|\cdot, \ldots, \cdot\|\right)$. But by (55), $x=$ $\left(x_{k}\right) \notin w_{\infty}^{\theta}\left(\mathscr{M}, B_{\wedge}^{\mu}, p,\|\cdot, \ldots, \cdot\|\right)$ which contradicts (ii). Hence (iii) must hold.

(iii) $\Rightarrow$ (i) Let (iii) hold. Suppose that $x=\left(x_{k}\right) \notin w_{\infty}^{\theta}(\mathscr{M}$, $\left.B_{\wedge}^{\mu}, p,\|\cdot, \ldots, \cdot\|\right)$. Then for $x=\left(x_{k}\right) \in w_{\infty}^{\theta}\left(B_{\wedge}^{\mu}, p,\|\cdot, \ldots, \cdot\|\right)$.

$$
\sup _{r} \frac{1}{h_{r}} \sum_{k \in I_{r}} M_{k}\left(\left\|\left(\frac{B_{\wedge}^{\mu} x_{k}}{\rho}, z_{1}, \ldots, z_{n-1}\right)\right\|\right)^{p_{k}}=\infty .
$$

Let $t=\left\|B_{\wedge}^{\mu} x_{k} / \rho, z_{1}, \ldots, z_{n-1}\right\|$ for each $k$, and then by (57) $\sup _{r}\left(1 / h_{r}\right) \sum_{k \in I_{r}} M_{k}(t)^{p_{k}}=\infty$, which contradicts (iii). Hence (i) must hold. This completes the proof of the theorem.

Theorem 7. Let $\mathscr{M}=\left(M_{k}\right)$ be a Musielak-Orlicz function. Then the following statements are equivalent:

(i) $w_{0}^{\theta}\left(\mathscr{M}, B_{\wedge}^{\mu}, p,\|\cdot, \ldots, \cdot\|\right) \subset w_{0}^{\theta}\left(B_{\wedge}^{\mu}, p,\|\cdot, \ldots, \cdot\|\right)$;

(ii) $w_{0}^{\theta}\left(\mathscr{M}, B_{\wedge}^{\mu}, p,\|\cdot, \ldots, \cdot\|\right) \subset w_{\infty}^{\theta}\left(B_{\wedge}^{\mu}, p,\|\cdot, \ldots, \cdot\|\right)$;

(iii) $\inf _{r}\left(1 / h_{r}\right) \sum_{k \in I_{r}} M_{k}(t)^{p_{k}}>0$ for all $t>0$.

Proof. (i) $\Rightarrow$ (ii) is obvious

(ii) $\Rightarrow$ (iii) Let (ii) hold and let (iii) fail to hold. Then

$$
\inf _{r} \frac{1}{h_{r}} \sum_{k \in I_{r}} M_{k}(t)^{p_{k}}=0 \quad \text { for some } t>0,
$$

and we can find a subinterval $I_{r(m)}$ of the set of intervals $I_{r}$ such that

$$
\frac{1}{h_{r}(m)} \sum_{k \in I_{r(m)}} M_{k}(m)^{p_{k}}<\frac{1}{m}, \quad m=1,2,3, \ldots
$$

Let us define $x=\left(x_{k}\right)$ as follows:

$$
B_{\wedge}^{\mu} x_{k}= \begin{cases}\rho m, & k \in I_{r(m)} \\ 0, & k \notin I_{r(m)} .\end{cases}
$$

Thus by (iii), $x=\left(x_{k}\right) \in w_{0}^{\theta}\left(\mathscr{M}, B_{\wedge}^{\mu}, p,\|\cdot, \ldots, \cdot\|\right)$. But $x=$ $\left(x_{k}\right) \notin w_{\infty}^{\theta}\left(B_{\wedge}^{\mu}, p,\|\cdot, \ldots, \cdot\|\right)$ which contradict (ii). Hence (iii) must hold.

(iii) $\Rightarrow$ (i) Let (iii) hold. Suppose that $x=\left(x_{k}\right) \in$ $w_{0}^{\theta}\left(\mathscr{M}, B_{\wedge}^{\mu}, p,\|\cdot, \ldots, \cdot\|\right)$. Therefore,

$$
\frac{1}{h_{r}} \sum_{k \in I_{r}} M_{k}\left(\left\|\left(\frac{B_{\wedge}^{\mu} x_{k}}{\rho}, z_{1}, \ldots, z_{n-1}\right)\right\|\right)^{p_{k}} \longrightarrow 0 \quad \text { as } r \longrightarrow \infty .
$$

Again suppose $x=\left(x_{k}\right) \notin w_{0}^{\theta}\left(B_{\wedge}^{\mu}, p,\|\cdot, \ldots, \cdot\|\right)$ for some number $\varepsilon>0$ and a subinterval $I_{r(m)}$ of the set of intervals $I_{r}$, we have

$$
\left\|\left(\frac{B_{\wedge}^{\mu} x_{k}}{\rho}, z_{1}, \ldots, z_{n-1}\right)\right\| \geq \varepsilon \quad \forall k
$$

Then, from properties of the Orlicz function, we can write

$$
M_{k}\left(\left\|\left(\frac{B_{\wedge}^{\mu} x_{k}}{\rho}, z_{1}, \ldots, z_{n-1}\right)\right\|\right)^{p_{k}} \geq M_{k}(\varepsilon)^{p_{k}} .
$$

Consequently, by (61), we have $\lim _{r \rightarrow \infty}\left(1 / h_{r}\right) \sum_{k \in I_{r}} M_{k}$ $(\varepsilon)^{p_{k}}=0$, which contradicts (iii). Hence (i) must hold. This completes the proof of the theorem.

Theorem 8. (i) If $0<\inf p_{k} \leq p_{k} \leq 1$ for all $k$, then $w^{\theta}\left(\mathscr{M}, B_{\wedge}^{\mu},\|\cdot, \ldots, \cdot\|\right) \subseteq w^{\theta}\left(\mathscr{M}, B_{\wedge}^{\mu}, p,\|\cdot, \ldots, \cdot\|\right)$.

(ii) If $1 \leq p_{k} \leq \sup _{k}=H<\infty$, then $w^{\theta}\left(\mathscr{M}, B_{\wedge}^{\mu}, p,\|\cdot, \ldots, \cdot\|\right) \subseteq w^{\theta}\left(\mathscr{M}, B_{\wedge}^{\mu},\|\cdot, \ldots, \cdot\|\right)$.

Proof. (i) Let $x \in w^{\theta}\left(\mathscr{M}, B_{\wedge}^{\mu},\|\cdot, \ldots, \cdot\|\right)$. Since $0<\inf p_{k} \leq 1$, we get

$$
\begin{aligned}
& \frac{1}{h_{r}} \sum_{k \in I_{r}} M_{k}\left(\left\|\left(\frac{B_{\wedge}^{\mu} x_{k}-L}{\rho}, z_{1}, \ldots, z_{n-1}\right)\right\|\right) \\
& \quad \leq \frac{1}{h_{r}} \sum_{k \in I_{r}} M_{k}\left(\left\|\left(\frac{B_{\wedge}^{\mu} x_{k}-L}{\rho}, z_{1}, \ldots, z_{n-1}\right)\right\|\right)^{p_{k}},
\end{aligned}
$$

and hence $x \in w^{\theta}\left(\mathscr{M}, B_{\wedge}^{\mu}, p,\|\cdot, \ldots, \cdot\|\right)$.

(ii) $1 \leq p_{k} \leq \sup p_{k}=H<\infty$ and $x=\left(x_{k}\right) \epsilon$ $w^{\theta}\left(\mathscr{M}, B_{\wedge}^{\mu}, p,\|\cdot, \ldots, \cdot\|\right)$. Then for each $0<\varepsilon<1$ there exists a positive integer $s_{0}$ such that

$$
\begin{gathered}
\frac{1}{h_{r}} \sum_{k \in I_{r}} M_{k}\left(\left\|\left(\frac{B_{\wedge}^{\mu} x_{k}-L}{\rho}, z_{1}, \ldots, z_{n-1}\right)\right\|\right)^{p_{k}} \\
\leq \varepsilon<1 \quad \forall r>s_{0} .
\end{gathered}
$$

This implies that

$$
\begin{aligned}
& \frac{1}{h_{r}} \sum_{k \in I_{r}} M_{k}\left(\left\|\left(\frac{B_{\wedge}^{\mu} x_{k}-L}{\rho}, z_{1}, \ldots, z_{n-1}\right)\right\|\right)^{p_{k}} \\
& \quad \leq \frac{1}{h_{r}} \sum_{k \in I_{r}} M_{k}\left(\left\|\left(\frac{B_{\wedge}^{\mu} x_{k}-L}{\rho}, z_{1}, \ldots, z_{n-1}\right)\right\|\right) .
\end{aligned}
$$

Therefore $x=\left(x_{k}\right) \in w^{\theta}\left(\mathscr{M}, B_{\wedge}^{\mu},\|\cdot, \ldots, \cdot\|\right)$. This completes the proof of the theorem.

\section{Conflict of Interests}

The authors declare that there is no conflict of interests regarding the publication of this paper.

\section{Acknowledgments}

The authors gratefully acknowledge that this research was partially supported by the University Putra Malaysia under the ERGS Grant Scheme having Project no. ERGS 1$2013 / 5527179$. The authors are grateful also to the anonymous referees for a careful checking of the details and for helpful comments that improved the paper. 


\section{References}

[1] H. Kizmaz, "On certain sequence spaces," Canadian Mathematical Bulletin, vol. 24, no. 2, pp. 169-176, 1981.

[2] M. Et and R. Çolak, "On some generalized difference sequence spaces," Soochow Journal of Mathematics, vol. 21, no. 4, pp. 377386, 1995.

[3] M. Et and M. Basarir, "On some new generalized difference sequence spaces," Periodica Mathematica Hungarica, vol. 35, no. 3, pp. 169-175, 1997.

[4] H. Dutta, "On some differece sequences spaces," Pacific Journal of Science and Technology, vol. 10, pp. 243-247, 2009.

[5] H. Dutta, "Some statistically convergent difference sequence spaces defined over real 2-normed linear spaces," Applied Sciences, vol. 12, pp. 37-47, 2010.

[6] M. Işik, "On statistical convergence of generalized difference sequences," Soochow Journal of Mathematics, vol. 30, no. 2, pp. 197-205, 2004.

[7] M. Mursaleen, "Generalized spaces of difference sequences," Journal of Mathematical Analysis and Applications, vol. 203, no. 3, pp. 738-745, 1996.

[8] M. Mursaleen, V. Karakaya, H. Polat, and N. Simşek, "Measure of noncompactness of matrix operators on some difference sequence spaces of weighted means," Computers \& Mathematics with Applications, vol. 62, no. 2, pp. 814-820, 2011.

[9] M. Mursaleen and A. K. Noman, "On some new difference sequence spaces of non-absolute type," Mathematical and Computer Modelling, vol. 52, no. 3-4, pp. 603-617, 2010.

[10] M. Mursaleen and A. K. Noman, "Compactness of matrix operators on some new difference sequence spaces," Linear Algebra and Its Applications, vol. 436, no. 1, pp. 41-52, 2012.

[11] S. A. Mohiuddine and A. Alotaibi, "Some spaces of double sequences obtained through invariant mean and related concepts," Abstract and Applied Analysis, vol. 2013, Article ID 507950, 11 pages, 2013.

[12] S. A. Mohiuddine, K. Raj, and A. Alotaibi, "Some paranormed double difference sequence spaces for Orlicz functions and bounded-regular matrices," Abstract and Applied Analysis, vol. 2014, Article ID 419064, 10 pages, 2014.

[13] K. Raj, S. K. Sharma, and A. K. Sharma, "Some difference sequence spaces in $n$-normed spaces defined by MusielakOrlicz function," Armenian Journal of Mathematics, vol. 3, pp. 127-141, 2010.

[14] T. Bilgin, "Some new difference sequences spaces defined by an Orlicz function," Filomat, no. 17, pp. 1-8, 2003.

[15] F. Başar and B. Altay, "On the space of sequences of $p$ bounded variation and related matrix mappings," Ukrainian Mathematical Journal, vol. 55, no. 1, pp. 108-118, 2003.

[16] M. Başarir and M. Kayikçi, "On the generalized $B^{m}$-Riesz difference sequence space and $\beta$-property," Journal of Inequalities and Applications, vol. 2009, Article ID 385029, 18 pages, 2009.

[17] J. Lindenstrauss and L. Tzafriri, "On Orlicz sequence spaces," Israel Journal of Mathematics, vol. 10, pp. 379-390, 1971.

[18] L. Maligranda, Orlicz Spaces and Interpolation, Seminars in Mathematics, Polish Academy of Science, Warsaw, Poland, 1989.

[19] J. Musielak, Orlicz Spaces and Modular Spaces, vol. 1034 of Lecture Notes in Mathematics, Springer, Berlin, Germany, 1983.

[20] A. R. Freedman, J. J. Sember, and M. Raphael, "Some Cesarotype summability spaces," Proceedings of the London Mathematical Society, vol. 37, no. 3, pp. 508-520, 1978.
[21] S. Gähler, "2-metrische Räume und ihre topologische Struktur," Mathematische Nachrichten, vol. 26, pp. 115-148, 1963.

[22] A. Misiak, " $n$-inner product spaces," Mathematische Nachrichten, vol. 140, pp. 299-319, 1989.

[23] H. Gunawan, "On $n$-inner product, $n$-norms and the Cauchy Schwartz inequality," Scientiae Mathematicae Japonicae, vol. 5, pp. 47-54, 2001.

[24] H. Gunawan, "The space of $p$-summable sequences and its natural n-norm," Bulletin of the Australian Mathematical Society, vol. 64, no. 1, pp. 137-147, 2001.

[25] H. Gunawan and M. Mashadi, "On $n$-normed spaces," International Journal of Mathematics and Mathematical Sciences, vol. 27, no. 10, pp. 631-639, 2001.

[26] K. Raj, A. K. Sharma, and S. K. Sharma, "A sequence space defined by Musielak-Orlicz function," International Journal of Pure and Applied Mathematics, vol. 67, no. 4, pp. 475-484, 2011.

[27] K. Raj and S. K. Sharma, "Some sequence spaces in 2-normed spaces defined by Musielak-Orlicz function," Acta Universitatis Sapientiae. Mathematica, vol. 3, no. 1, pp. 97-109, 2011.

[28] M. Mursaleen, S. K. Sharma, and A. Kılıçman, "Sequence spaces defined by Musielak-Orlicz function over $n$-normed spaces," Abstract and Applied Analysis, vol. 2013, Article ID 364743, 10 pages, 2013.

[29] K. Raj and S. K. Sharma, "Some multiplier sequence spaces defined by a Musielak-Orlicz function in $n$-normed spaces," New Zealand Journal of Mathematics, vol. 42, pp. 45-56, 2012.

[30] M. Et, Y. Altin, B. Choudhary, and B. C. Tripathy, "On some classes of sequences defined by sequences of Orlicz functions," Mathematical Inequalities \& Applications, vol. 9, no. 2, pp. 335342, 2006.

[31] M. Gungor and M. Et, " $\Delta^{r}$-strongly almost summable sequences defined by Orlicz functions," Indian Journal of Pure and Applied Mathematics, vol. 34, no. 8, pp. 1141-1151, 2003.

[32] M. Mursaleen, R. Çolak, and M. Et, "Some geometric inequalities in a new Banach sequence space," Journal of Inequalities and Applications, vol. 2007, Article ID 86757, 6 pages, 2007.

[33] B. C. Tripathy and H. Dutta, "On some new paranormed difference sequence spaces defined by Orlicz functions," Kyungpook Mathematical Journal, vol. 50, no. 1, pp. 59-69, 2010. 


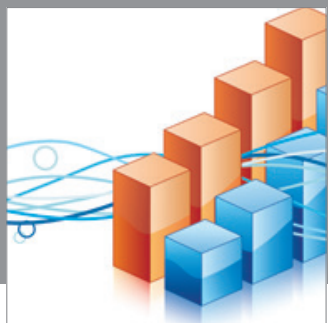

Advances in

Operations Research

mansans

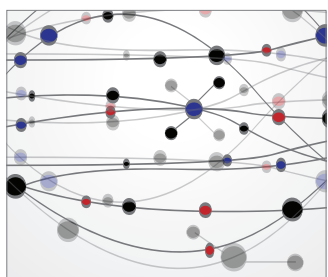

The Scientific World Journal
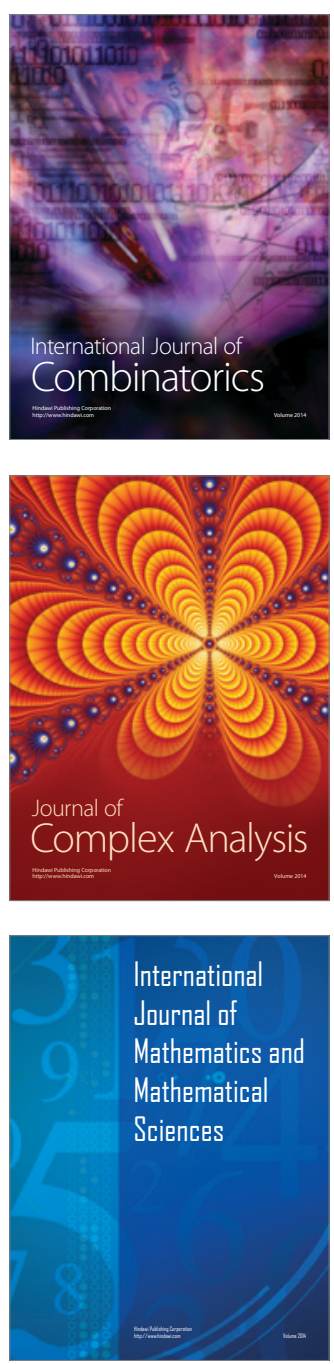
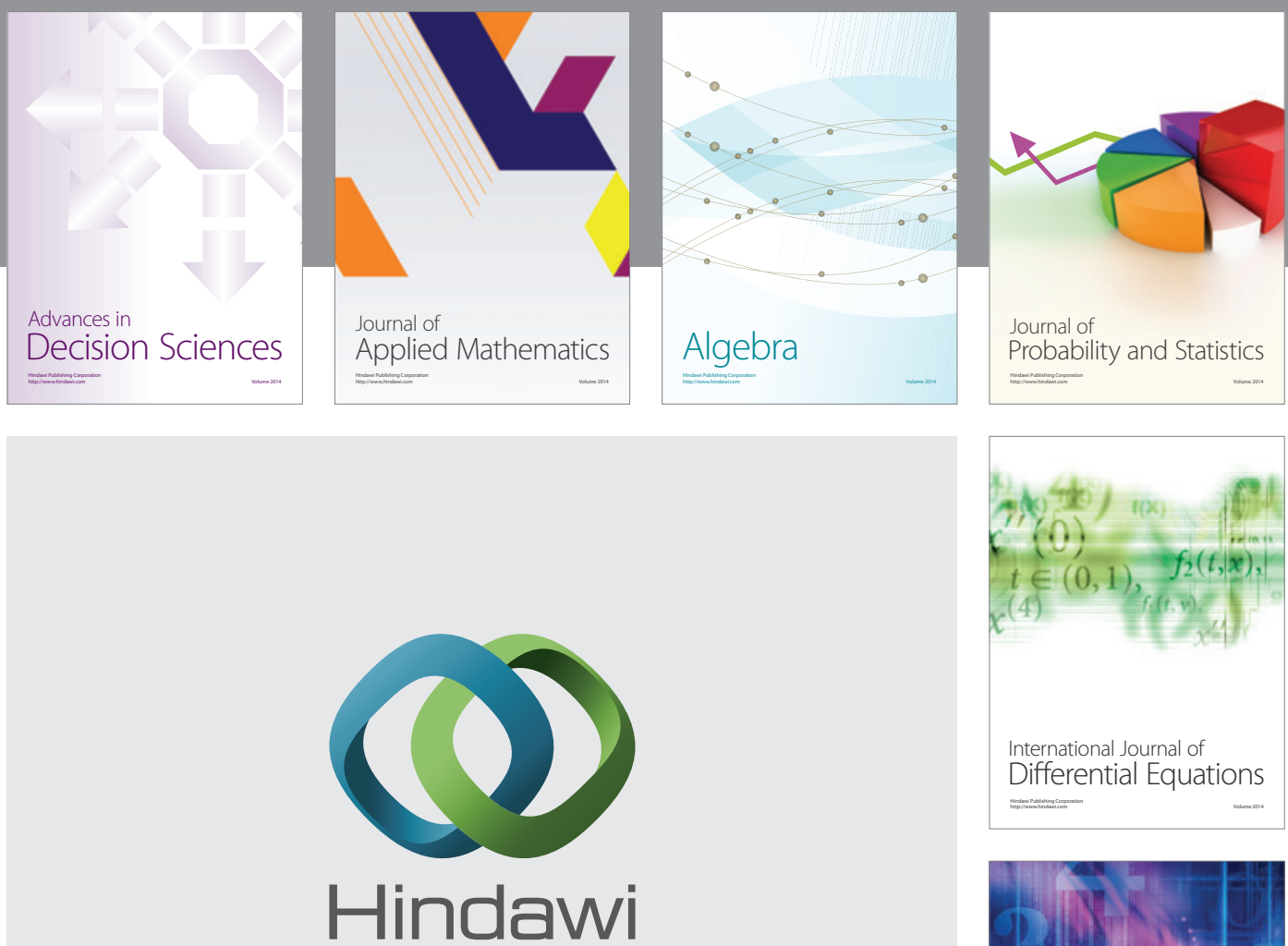

Submit your manuscripts at http://www.hindawi.com
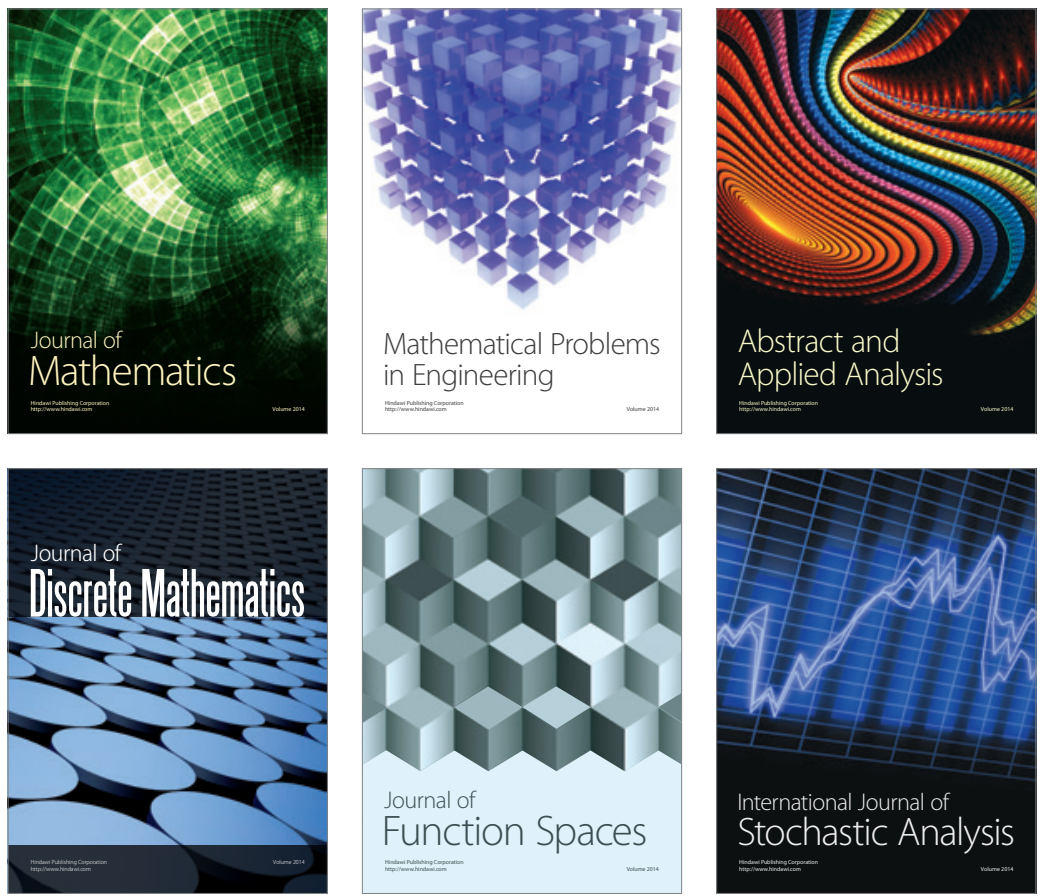

Journal of

Function Spaces

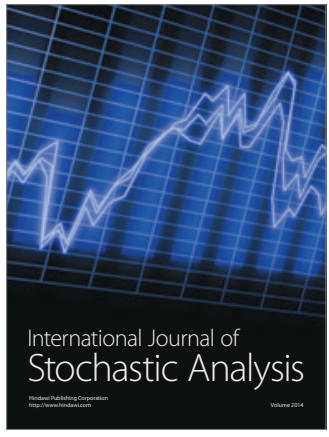

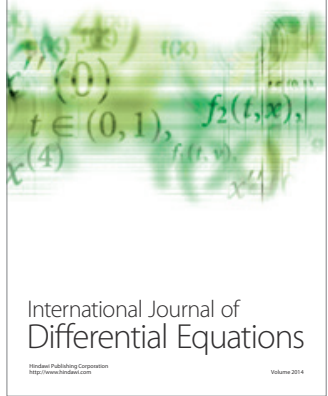
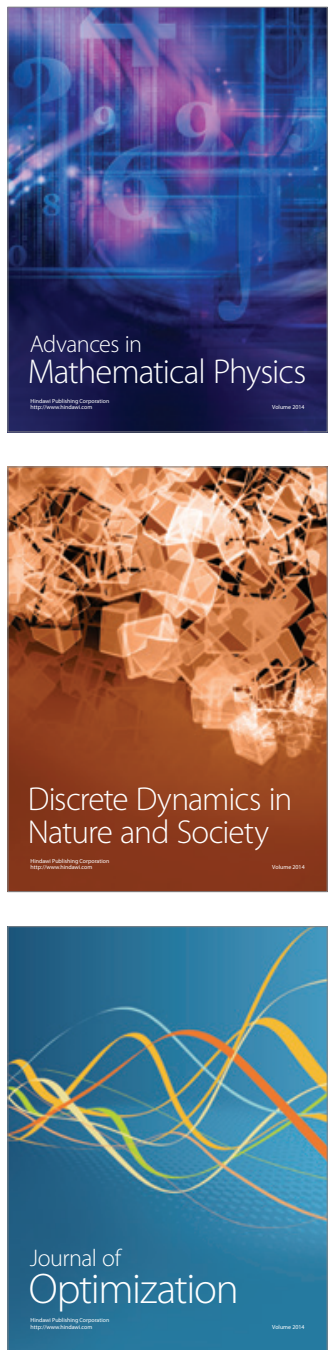